\title{
Characterization of Fam20C expression in odontogenesis and osteogenesis using transgenic mice
}

\author{
Er-Xia Du ${ }^{1}$, Xiao-Fang Wang ${ }^{2}$, Wu-Chen Yang ${ }^{1}$, Deborah Kaback ${ }^{3}$, Siu-Pok Yee ${ }^{3}$, Chun-Lin Qin ${ }^{2}$, Anne George $^{4}$ \\ and Jian-Jun Hao ${ }^{1}$
}

Our previous studies have demonstrated that Fam20C promotes differentiation and mineralization of odontoblasts, ameloblasts, osteoblasts and osteocytes during tooth and bone development. Ablation of the Fam20C gene inhibits bone and tooth growth by increasing fibroblast growth factor 23 in serum and causing hypophosphatemia in conditional knockout mice. However, control and regulation of the expression of Fam20C are still unknown. In this study, we generated a transgenic reporter model which expresses green fluorescence protein (GFP) driven by the Fam20C promoter. Recombineering was used to insert a 16 kb fragment of the mouse Fam 20C gene (containing the $15 \mathrm{~kb}$ promoter and $1.1 \mathrm{~kb}$ of exon 1) into a pBluescript SK vector with the topaz variant of GFP and a bovine growth hormone polyadenylation sequence. GFP expression was subsequently evaluated by histomorphometry on cryosections from E14 to adult mice. Fluorescence was evident in the bone and teeth as early as E17.5. The GFP signal was maintained stably in odontoblasts and osteoblasts until 4 weeks after birth. The expression of GFP was significantly reduced in teeth, alveolar bone and muscle by 8 weeks of age. We also observed colocalization of the GFP signal with the Fam20C antibody in postnatal 1- and 7-day-old animals. Successful generation of Fam20C-GFP transgenic mice will provide a unique model for studying Fam20Cgene expression and the biological function of this gene during odontogenesis and osteogenesis. International Journal of Oral Science (2014) 7, 89-94; doi:10.1038/ijos.2014.67; published 24 December 2014

Keywords: Fam20C; green fluorescence protein report; mouse; promoter

\section{INTRODUCTION}

Fam20C (also named dentin matrix protein 4) was initially identified in hematopoietic cells, and subsequently shown to be expressed in other tissues at various levels, including the liver and lung. ${ }^{1}$ Our previous study showed that Fam20C mRNA was detected at a high level in teeth and bone. ${ }^{2}$ Fam20C was highly expressed in odontoblasts, ameloblasts, cementoblasts, osteoblasts and osteocytes, as observed by in situ hybridization and immunohistochemistry. ${ }^{2-3}$ Overexpression of Fam20C in odontoblasts and osteoblasts promoted differentiation and mineralization of these cells. ${ }^{2}$ Mice with Fam20C specifically depleted in mineralized tissues developed hypophosphatemic rickets, and demonstrated a downregulation of phosphate and upregulation of fibroblast growth factor 23 in serum and bone. ${ }^{4}$ Fam20C was also shown to be critical for murine tooth development. ${ }^{4}$

Recent studies have shown that Fam20C was enriched in the Golgi fraction, possesses casein kinase activity and phosphorylates secretory pathway proteins. ${ }^{5-6}$ Mutations in Fam20C, which cause lethal osteosclerotic bone dysplasia (Raines syndrome), are due to the inability of Fam20C to effectively phosphorylate other bone and teeth matrix proteins. Patients carrying mutations in Fam20C are characterized by craniofacial anomalies including microcephaly, exophthalmos, midface hypoplasia, cleft palate, gingival hypertrophy and generalized osteosclerosis on radiography. ${ }^{7-8}$ Taken together, these data suggest that Fam20C plays a fundamental role in the development and maintenance of mineralized tissues. However, very little is known about the mechanisms regulating Fam20C expression. It is well established that the tissue-specific nature of Fam20C gene expression is largely regulated by various trans-acting factors interacting with cis-acting elements in the promoter regions of the gene. Therefore, analysis of the cis-regulatory regions represents an initial and necessary step in studying the regulation of the gene.

In the present study, we have generated transgenic mice expressing green fluorescence protein (GFP) under the control of the $16.1 \mathrm{~kb}$ Fam20C 5' upstream sequence, as a first step to characterize the spatiotemporal regulation of Fam20C during development. Our results clearly show that the Fam20C promoter can drive the specific expression of the GFP reporter in the teeth and other mineralized tissues. This transgenic model will allow us to further assess the expression pattern of Fam20C in vivo, as well as to isolate Fam20C-positive cells for further ex vivo studies.

\section{MATERIALS AND METHODS}

Generation of the Fam20C-GFP construct

Recombineering was used to insert a $16.1 \mathrm{~kb}$ fragment of the mouse Fam20C genomic region (consisting of the $15 \mathrm{~kb}$ promoter and $1.1 \mathrm{~kb}$

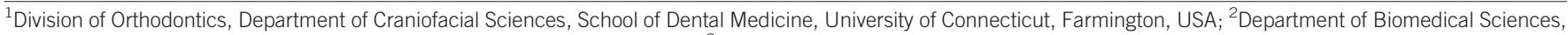

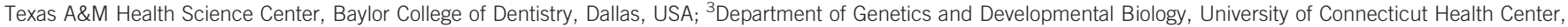
Farmington, USA and ${ }^{4}$ Department of Oral Biology, University of Illinois, Chicago, USA

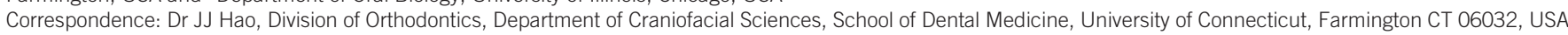
E-mail: Jhao@uchc.edu

Accepted 18 December 2013 
of the exon 1 sequence) into a pBluescript SK vector (Agilent Technologies, Santa Clara, CA, USA) with the topaz variant of GFP and bovine growth hormone polyadenylation sequence by gap repair. The $20.6 \mathrm{~kb}$ expression cassette was released from the construct backbone with NotI and SalI (New England Biolabs, Ipswich, MA, USA) digestion. The combination of the Fam20C promoter and GFP was sequenced to verify the construct.

\section{Transient transfection of the Fam20C-GFP construct}

The $20.6 \mathrm{~kb}$ Fam20C-GFP plasmid was transiently transfected into HEK293 and MC3T3 cells using Lipofectamine 2000 (Invitrogen, Grand island, NY, USA). In brief, these two cell lines were seeded onto $10 \mathrm{~cm}$ dishes ( $80 \%$ confluent). Ten microliters of Lipofectamine with $2 \mu \mathrm{g}$ construct was added to serum starved cells. The media was changed after $4 \mathrm{~h}$ with normal serum medium. As a control, the cells were transfected with the original empty pBluescript SK plasmid with a cytomegalovirus (CMV) promoter (Agilent Technologies, Santa Clara, CA, USA). The GFP signal was observed and imaged using a GFP filter cube on a Zeiss Axiovert 200M epifluorescence microscope (Zeiss, Jena, Germany) 48 h later.

\section{Generation of transgenic mice}

The 20.6 kb Fam20C-GFP (Topaz) construct was prepared for pronuclear injection by isolating the plasmid DNA by a Midiprep kit (Qiagen, Santa Clara, CA, USA). DNA was dissolved in injection buffer and dialyzed for $20 \mathrm{~min}$ against injection buffer using the Millipore 'drop dialysis' technique. At the UCONN Health Center Transgenic Mouse and Gene Targeting Core, the 16.6 kb Fam20C-GFP fragment was injected into pronuclei of fertilized eggs from C57BL6j embryos. The ovum was subsequently implanted into pseudopregnant females. Offspring were analyzed for insertion of the construct by polymerase chain reaction (PCR) amplification of tail DNA, using the transgenic specific genotyping primers for the Fam20C transgene forward: 5' CTGCTGCACCTGCCAGATCGTC-3', GFP reverse: 5'-GCTCGACCAGGATGGGCACC-3', which amplifies a 573 base-pair product across Fam20C and GFP. Four positive transgenic founder lines were fertile, and none exhibited extraneous phenotypes. One line was selected for further characterization based upon high GFP expression levels and the pattern of GFP signal in both tooth and bone.

\section{Frozen tissue sections}

To maintain consistency with previous publications, ${ }^{2-3}$ embryonic day 14.5 (E14.5), embryonic day 17.5 (E17.5), postnatal 1 day-old (P1), 7-day-old (P7) and 8-week-old mice were used to systematically analyze the expression of Fam20C in the teeth and surrounding tissues. The acquired tissues were fixed with $4 \%$ formalin in phosphatebuffered saline (PBS) solution at $4{ }^{\circ} \mathrm{C}$ overnight, and then transferred into $30 \%$ sucrose for an additional night. The head and mandibles were processed for optimal cutting temperature (OCT) compound embedding, and serial $6 \mu \mathrm{m}$ sections were prepared. The animal protocol was approved by the University of Connecticut Health Center Institutional Animal Use and Care Committee (100332-0115).

\section{Immunohistochemistry}

To further localize endogenous Fam20C expression in teeth, immunohistochemistry was performed in transgenic mice at P1 and P7. Staining was carried out as previously described. ${ }^{9}$ Briefly, the frozen sections were dried at room temperature for $30 \mathrm{~min}$, then rinsed in PBS for 10 min. Tissues were permeabilized with $0.5 \%$ Triton-X-100 (T8787; Sigma Chemical, St. Louis, MO, USA) in PBS for $30 \mathrm{~min}$ at room temperature. Sections were washed two times for $10 \mathrm{~min}$ in PBS, and incubated with Power Block (Kirkegaard and Perry Laboratories, Gaithersburg, MD, USA) for $20 \mathrm{~min}$ at room temperature. Power Block reagent (with casein) contains a proprietary, synergistic, antioxidant sequestering agent which removes trace metals and oxidizers that could adversely affect immune detection sensitivity and specificity. Sections were then rinsed in PBS twice and incubated with a Fam20C polyclonal antibody $\left(8 \mu \mathrm{g} \mathrm{IgG} \cdot \mathrm{mL}^{-1}\right)$ overnight at $4^{\circ} \mathrm{C}$. Fam $20 \mathrm{C}$ antibody (generously provided by Dr Chunlin Qin) was diluted in PBS containing $1 \%$ bovine serum albumin (BSA) and $1 \%$ goat serum (Sigma Chemical, St. Louis, MO, USA). After washing three times in PBS for $10 \mathrm{~min}$, the sections were incubated at room temperature for $2 \mathrm{~h}$ with goat anti-rabbit immunoglobulin $\mathrm{G}(\mathrm{IgG})(\mathrm{H}+\mathrm{L})$ secondary antibody (red-fluorescent Alexa Fluor 594 dye, $2 \mathrm{mg} \cdot \mathrm{mL}^{-1}$; Life Technologies, Grand Island, NY, USA) which was diluted 500 times in $1 \% \mathrm{BSA}$ and $1 \%$ goat serum in PBS. The sections were washed 3 times in PBS for $10 \mathrm{~min}$. 4',6-diamidino-2-phenylindole (DAPI) (Sigma Chemical, St. Louis, MO, USA) was used to stain the nuclei.

\section{RESULTS}

\section{Conserved non-coding sequences between human and mouse Fam20C genes}

Islands of conserved regions between mouse and human Fam20C genes were identified using Vista plots software (http://wwwgsd.lbl.gov/vista/ and zpicture.dcode.org/). The dentin matrix acidic phosphoprotein 1 (DMP1), which is critical for proper mineralization of bone and dentin, is also present in diverse cells of bone and tooth tissues. ${ }^{10-11}$ Previous experiments using this method with DMP1 have shown that the non-coding islands of sequence conservation have a significantly increased probability of playing a role in gene regulation or other functions in vivo. ${ }^{12-13}$ Three of the highly conserved DNA regions were observed in the first $15 \mathrm{~kb}$ of the Fam20C promoter region, as shown in Figure 1a. Based on this information, we chose to analyze the first $15 \mathrm{~kb}$ of the $5^{\prime}$-flanking region, and used a GFP reporter for monitoring cis-regulatory activity in odontogenesis and osteogenesis.

\section{Tests of the Fam20C-GFP construct}

The transgene construct of Fam20C-GFP was transiently transfected in HEK293 and MC3T3 cells. It was clearly shown that there were GFP-positive cells in the HEK293 cells (with an efficiency of approximately $15 \%$; Figure $2 \mathrm{c}$ and $2 \mathrm{~d}$ ). However, there is weaker signal observed in the MC3T3 cells (Figure $2 \mathrm{~g}$ and $2 \mathrm{~h}$ ). The positive controls consisted of cells transfected with a GFP construct under the transcriptional control of a CMV promoter in the pBluescript SK vector (Figure 2a, 2b, 2e and 2f). Although the efficiency was lower in cells transfected with the transgene construct than in control cells, the expression of the GFP signal in MC3T3 cells demonstrated that the Fam20C-GFP construct could be successfully transferred into osteoblast cell nucleus.

\section{Generation of transgenic mouse lines}

Four positive transgenic founder lines were derived by pronuclear injection of the Fam20C-GFP construct into fertilized ova from C57BL6j mice. All the founder mice that were PCR-positive for the transgene developed normally and had no aberrant phenotype that was detectable by 12 months of age. We chose to maintain one line, based on their consistent and robust expression of GFP-positive signal in odontoblasts and osteoblasts during development. 
a Mouse
vs
Human

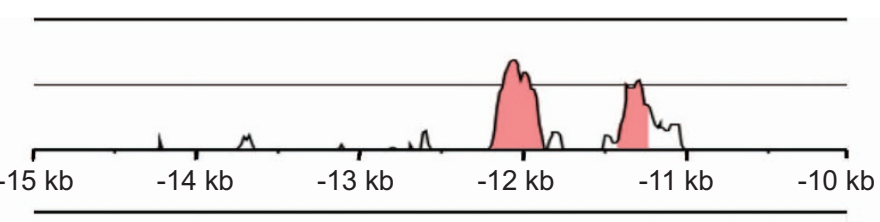

$100 \%$

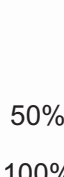

Mouse

vs
Human

Mouse

Mouse

vs

Human
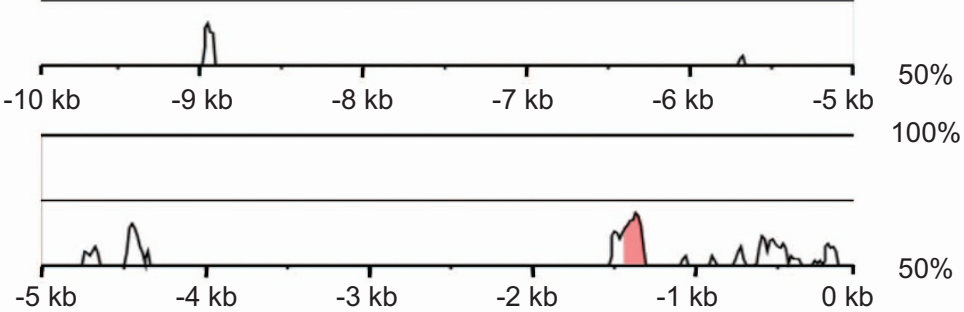

b Notl

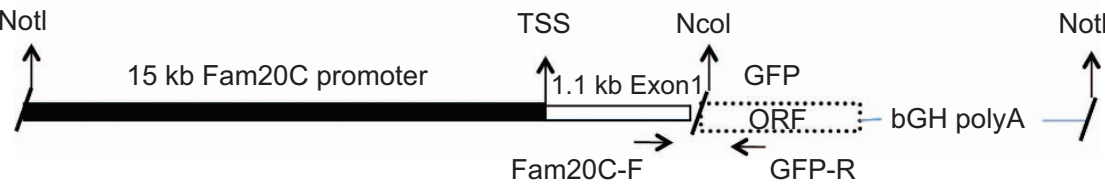

Figure 1 Strategy for generation of Fam20C-GFP. (a) Candidate cis-regulatory regions in the Fam20C gene. Conserved non-coding sequence islands are found by comparing the mouse and human Fam20C genes. Many of the conserved non-coding sequences are in the first $15 \mathrm{~kb}$ of the 5'-promoter. The scale on the $y$-axis goes from $50 \%$ to $100 \%$ homology. The three red regions are peaks of conserved nucleotide sequences with a minimum of $70 \%$ homology. (b) Schematic diagram of the Fam20C-GFP construct. The $20.6 \mathrm{~kb}$ construct contains the $15 \mathrm{~kb}$ Fam20C 5'-promoter, $1.1 \mathrm{~kb}$ of exon 1, GFP (topaz) and bGHpolyA. bGHpolyA, bovine growth hormone polyadenylation sequence; GFP, green fluorescence protein.

\section{Developmental expression of Fam20C-GFP}

The expression profiles of GFP were systematically examined in the incisors and molars of the mouse mandible. In order to remain consistent with previous publications, ${ }^{2-3}$ we focused on the results in lower incisors and upper molars as well as their surrounding tissues during odontogenesis and osteogenesis.

GFP was not observed in any odontogenic, chondrogenic and osteogenic tissues at E14.5 (data not shown). At E17.5, GFP was detected in the osteoblasts at the ossification sites of the craniofacial bones such as calvarial, nasal and alveolar bones. When the lower incisors reached their late cap stage at E17.5, GFP was shown in the secretory odontoblasts in the tooth and osteoblasts of alveolar bone (Figure 3).
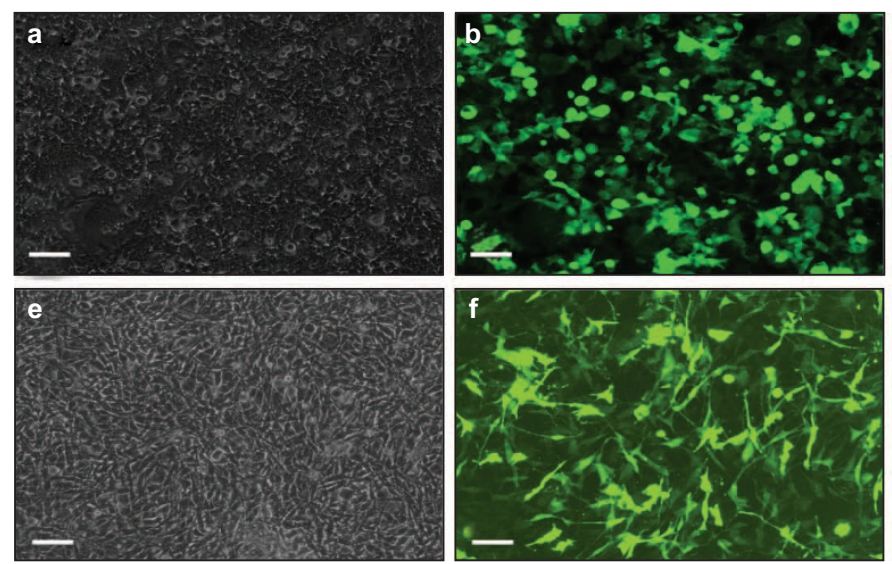

The intensity of the GFP signal increased dramatically, and was specifically restricted in the odontoblasts and osteoblasts of newborn P1 transgenic mice (Figure 4). At this stage, GFP was highly expressed in new secretory odontoblasts and osteoblasts. GFP expression was shown clearly and specifically in the cranial base as well as the calvarial, nasal, alveolar bones. To ensure that our transgene was correctly expressed in mineralized tissues, we stained heads from the transgenic line with an anti-Fam20C antibody. This analysis was performed in frozen sections at P1, where GFP allowed for a clear indication of overlapping localization of Fam20C antibody staining (Figure 4d).

The GFP signal continued to increase in odontoblasts and osteoblasts in P7 mice (Figure 5). The intense GFP signal was maintained stably in incisors and molars until 4 weeks after birth (data not shown).

Figure 2 The Fam20C-GFP construct shows GFP expression in HEK293 cell line and MC3T3 cell line after transient transfection. (c, d) HEK293 cell line; (g, h) MC3T3 cell line. (a, $\mathbf{c}, \mathbf{e}$ and $\mathbf{g}$ ) Bright field; (b, d, $\mathbf{f}$ and $\mathbf{h}$ ) fluorescence. $\mathbf{a}, \mathbf{b}, \mathbf{e}$ and $\mathbf{f}$ are the controls. $\mathbf{c}, \mathbf{d}, \mathbf{g}$ and $\mathbf{h}$ are the cells transiently transfected with Fam20C-GFP construct. Bar $=200 \mu \mathrm{m}$. GFP, green fluorescence protein. 

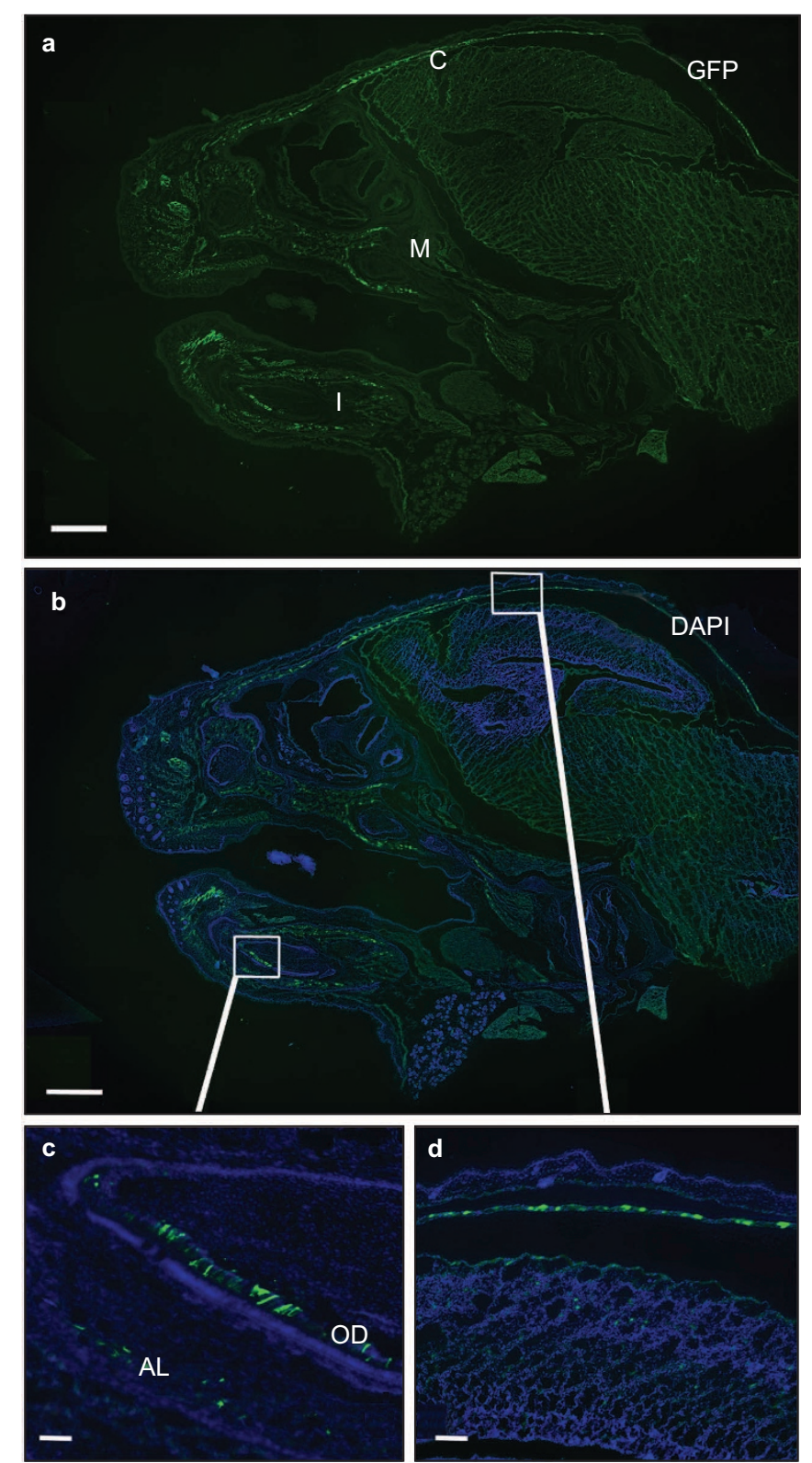

Figure 3 Identification of GFP in the head of E17.5 transgenic mice. Heads were collected from E17.5 mice, fixed, embedded and cut into $6 \mu \mathrm{m}$ cryosections. GFP was expressed in secretory odontoblasts of the incisors and osteoblasts of the calvarias, alveolar and nasal bones. (a) GFP; (b) GFP and DAPI; (c, d) the boxed areas in $\mathbf{b}$. Bar in $\mathbf{a}$ and $\mathbf{b}, 500 \mu \mathrm{m}$; bar in $\mathbf{c}$ and d, $200 \mu \mathrm{m}$. AL, alveolar; C, calvarias; DAPI, 4',6-diamidino-2-phenylindole; E17.5, embryonic day 17.5; green fluorescence protein; I, incisor; M, molar; OD, odontoblasts.

There was also visible GFP signal in calvarial, nasal, alveolar bones and the cranial base in P7 transgenic mice. Immunostaining clearly showed that the Fam20C antibody almost completely overlapped with the GFP signal in odontoblasts and osteoblasts at P7 (Figure 5d and 5e). Fam20C antibody and GFP were not visible in ameloblasts at this stage.

The expression of GFP in the incisors and molars decreased with age, starting around 4 weeks, and was nearly undetectable by 8 weeks in the molars (Figure 6). However, the GFP signal in the incisors and first molars remained at a low level in 8-week-old mice. Alveolar bone
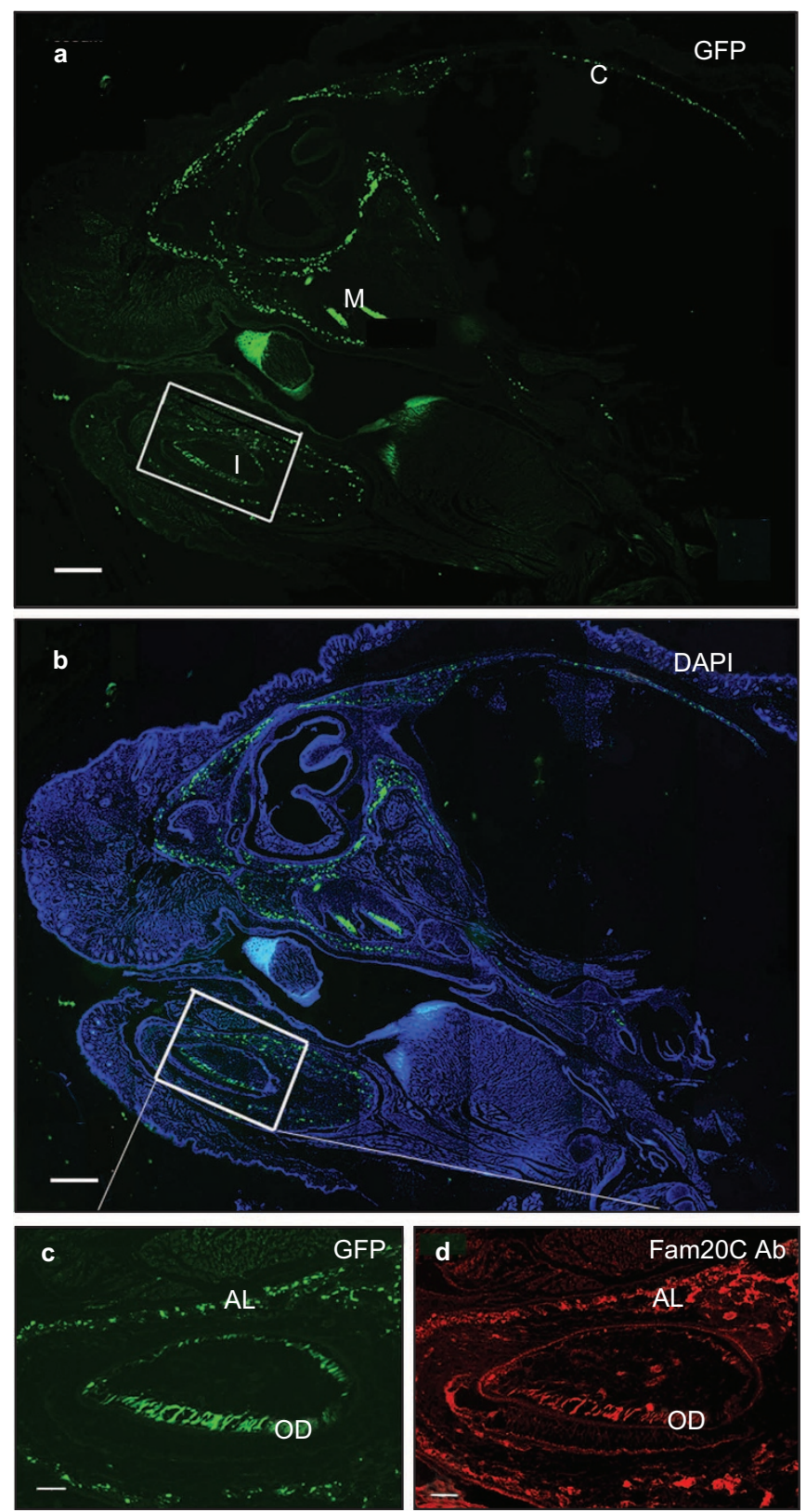

Figure 4 Identification of GFP in the head of P1 transgenic mice. GFP expression level progressively increased in the secretory odontoblasts of the incisors and upper first molars and osteoblasts of calvarias, alveolar and nasal bones. (a) GFP. (b) GFP and DAPI; (c and d) the boxed areas in b. (c) GFP fluorescence. (d) Fam20C antibody staining with FITC secondary antibody. Bar in $\mathbf{a}$ and $\mathbf{b}$, $500 \mu \mathrm{m}$; bar in c and d, $200 \mu \mathrm{m}$. AL, alveolar; C, calvarias; DAPI, 4',6-diamidino-2-phenylindole; FITC, fluorescein isothiocyanate; GFP, green fluorescence protein; I, incisor; M, molar; OD, odontoblasts; P1, postnatal 1 day-old.

in proximity to the first molars showed weak GFP signal (Figure 6). The muscle and other soft tissues also expressed GFP at a low level (Figure 6). This is consistent with what was previously reported, that Fam20C mRNA was expressed in skeletal muscle and non-mineralized tissues in adult mice. ${ }^{1}$

The Fam20C-GFP mice had no apparent abnormalities throughout the 12-month time course examined. We have conservatively concluded that the Fam20C-GFP transgene does not have a major effect on tooth and bone development in this model. 

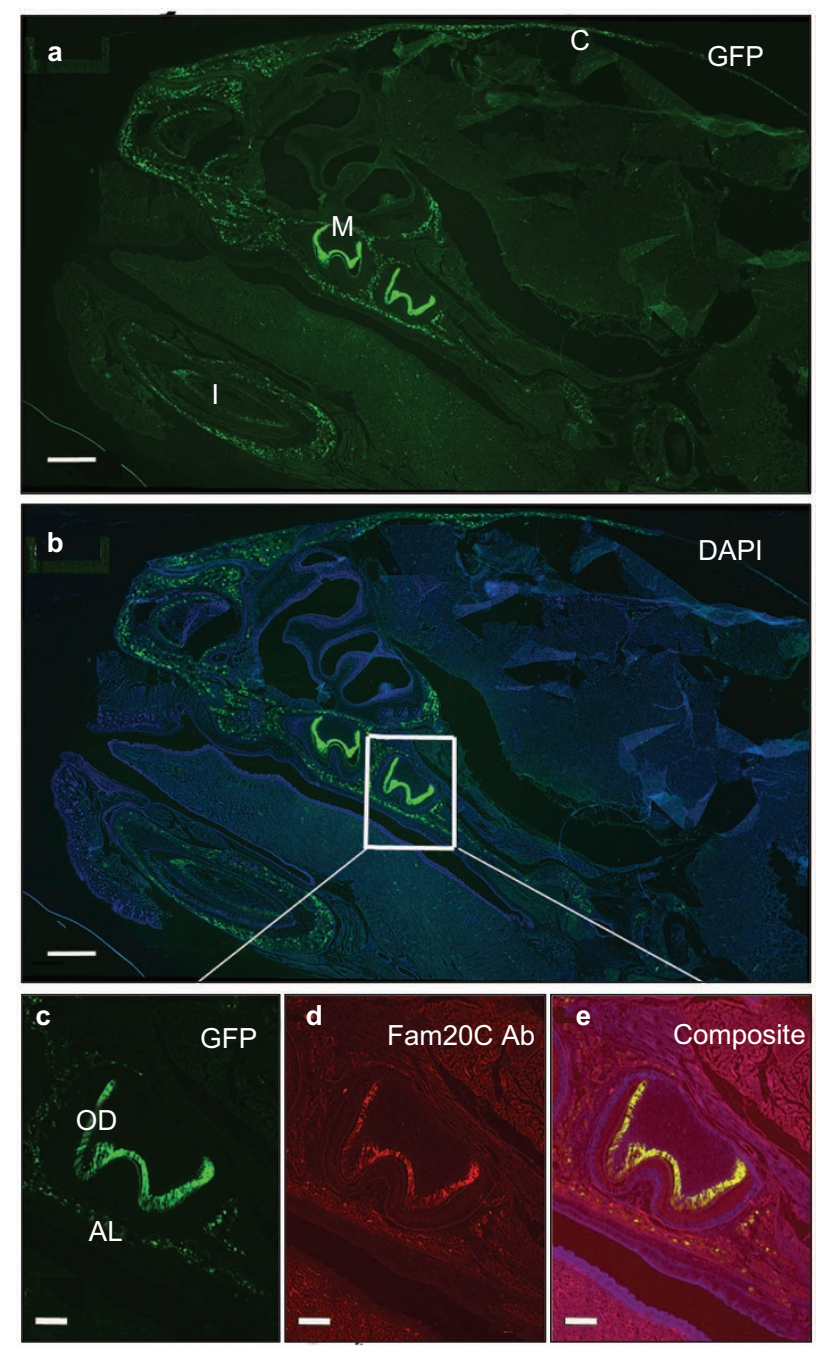

Figure 5 Identification of GFP in the head of P7 transgenic mice. GFP expression level significantly increased in the secretory odontoblasts of the first and second molars. GFP fluorescence was predominantly observed in the odontoblasts of the incisors, molars and osteoblasts of the calvaria, alveolar and nasal bones. (a) GFP; (b) GFP and DAPI; (c, $\mathbf{d}$ and $\mathbf{e}$ ) represent the boxed areas in b. (c) GFP fluorescence. (d) Fam20C antibody staining with FITC secondary antibody. (e) composite image of c and d. Bar in a and b, $500 \mu \mathrm{m}$; bar in c, d and e, $200 \mu \mathrm{m}$. AL, alveolar; C, calvarias; DAPI, 4',6-diamidino-2-phenylindole; GFP, green fluorescence protein; I, incisor; M, molar; OD, odontoblasts; P7, postnatal 7 day-old.

\section{DISCUSSION}

Fam20C is a calcium-binding protein kinase that specifically phosphorylates secreted proteins. ${ }^{5-6}$ The focus of this research was to evaluate the 15 - $\mathrm{kb}$ promoter region controlling Fam20C gene expression using a transgenic mouse model. A question of particular importance regarding the generation of a transgenic line is whether the expression pattern of the transgene reliably parallels the expression pattern of the endogenous gene, in this case Fam20C. We clearly verified that the expression pattern of GFP was very similar to that of endogenous Fam20C in odontoblasts and osteoblasts of P1 and P7 transgenic mice with a Fam20C polyclonal antibody (Figures 4 and 5). Interestingly, we also found the GFP signal was slightly offset from Fam20C signal in two different areas (Figures 4 and 5). First, GFP was not expressed in ameloblasts in which endogenous Fam20C is known to be expressed..$^{2-3}$ Second, the GFP signal was not detectable at E14.5 in BL6 transgenic mice, although it was previously demonstrated that Fam20C was expressed as early as E14.5 in the CD1 mouse strain. ${ }^{3}$ Thus, the fidelity of GFP expression in our transgenic mice did not completely match that of wild type mice. Although this discrepancy might be explained by differing genetic backgrounds of the two strains, we cannot assume that the $15-\mathrm{kb}$ promoter region used in our studies contains all the elements regulating the Fam20C gene. It is highly possible that the $15-\mathrm{kb}$ promoter lacks the critical region necessary for the regulation of Fam20C in ameloblasts.

We have successfully generated four founder lines in this study. The expression pattern and level of the GFP signal did not show any significant differences amongst the transgenic lines, suggesting that the expression of the transgene was not affected by position of insertion in the mouse genome. There were no observable abnormalities in these mice up through one year of age, suggesting the absence of major defects in cell function caused by the insertion of the GFP.

We have shown that the Fam20C-GFP signal was visible in the heads and mandibular areas analyzed at embryonic, postnatal and adult stages in mouse. We have not completely characterized the expression level and patterns of GFP in any other organs at this time. It will be of interest to characterize the GFP expression pattern in Fam20C-GFP transgenic mice during kidney development because it would provide the basis for studying hypophosphatemia and hyperphosphaturia in patients with Raine syndrome. ${ }^{14}$

In the future, we can trace GFP-positive cell lineages in this reporter model during odontogenesis and osteogenesis. GFP-positive cells can also be sorted through flow cytometry, and be used for dentin and bone regeneration. In addition, the Fam20C-GFP transgenic mouse is
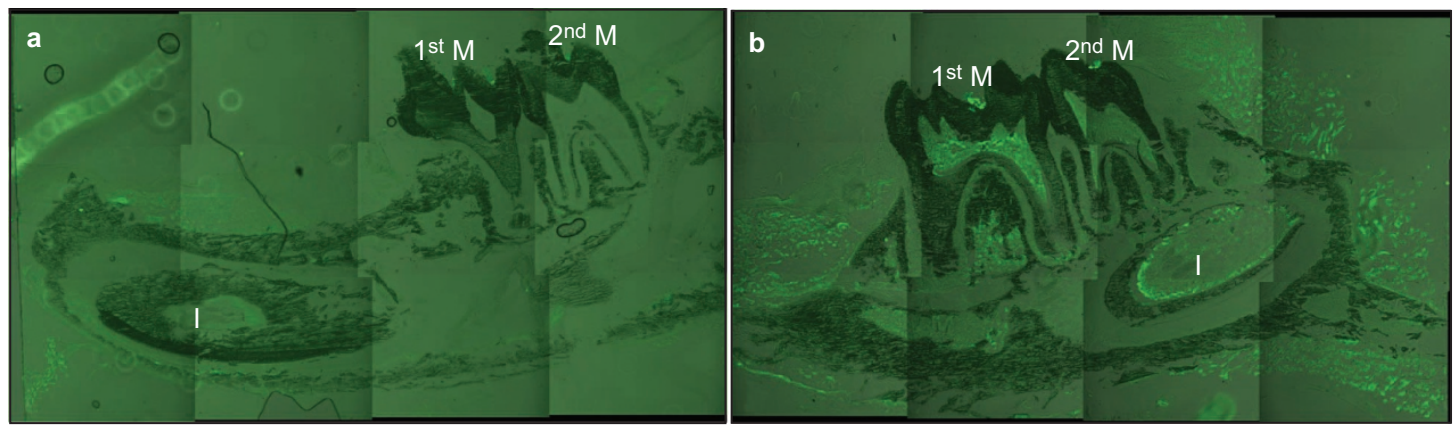

Figure 6 Identification of GFP in the head of postnatal 8-week-old transgenic mice. The GFP expression level is significantly reduced in the mature odontoblasts of tooth and in the osteoblasts of alveolar bones in adult mice. (a) Wild-type mice; (b) Fam20C-GFP transgenic mice. Bar in a and b, $500 \mu \mathrm{m}$. GFP, green fluorescence protein; I, incisor; M, molar. 
a suitable animal model for exploring orthodontic tooth movement because Fam20C is also expressed in osteocytes that are important mechanical sensors.

In conclusion, we have generated and characterized Fam20C-GFP reporter mice with respect to mineralized tissues. This study developed a novel mouse model, with which we are able to identify when and where Fam20C is expressed in different tissues and organs during development and in adulthood. After thorough characterization, this mouse model could be become a critical tool for studying the role of Fam20C in the development of skeletal tissues and disorders of phosphate homeostasis. Fam20C-GFP-positive cell lineages would offer a therapeutic approach for dentin and bone defects in clinical settings.

\section{ACKNOWLEDGEMENTS}

This work was supported by UCONN Health Center Startup Fund (Jian-Jun Hao) and the American Association of Orthodontists Foundation (AAOF) (Jian-Jun Hao). We thank Drs Mina Mina and David Rowe for their advice, guidance and discussion. We appreciate Dr Anne Delany and Sara Strecker for proofreading the manuscript. We acknowledge Dr Xi Jiang for assisting with fluorescence microscopy and frozen tissue sections.

1 Nalbant D, Youn $\mathrm{H}$, Nalbant SI et al. FAM20: an evolutionarily conserved family of secreted proteins expressed in hematopoietic cells. BMC Genomics 2005; 6: 11 .

2 Hao J, Narayanan K, Muni T et al. Dentin matrix protein 4, a novel secretory calciumbinding protein that modulates odontoblast differentiation. J Biol Chem 2007; 282(21): 15357-15365.

3 Wang X, Hao J, Xie $Y$ et al. Expression of FAM2OC in the osteogenesis and odontogenesis of mouse. J Histochem Cytochem 2010; 58(11): 957-967.
4 Wang X, Wang S, Li C et al. Inactivation of a novel FGF23 regulator, FAM20C, leads to hypophosphatemic rickets in mice. PLoS Genet 2012; 8(5): e1002708.

5 Tagliabracci VS, Engel JL, Wen J et al. Secreted kinase phosphorylates extracellular proteins that regulate biomineralization. Science 2012; 336(6085): 1150-1153.

6 Ishikawa HO, Xu A, Ogura E et al. The Raine syndrome protein FAM20C is a Golgi kinase that phosphorylates bio-mineralization proteins. PLoS One 2012; 7(8): e42988.

7 Raine J, Robertson ME, Malcolm S et al. Absence of $Y$ specific DNA sequences in two siblings with 46XX hermaphroditism. Arch Dis Child 1989; 64(8): 1185-1187.

8 Simpson MA, Hsu R, Keir LS et al. Mutations in FAM20C are associated with lethal osteosclerotic bone dysplasia (Raine syndrome), highlighting a crucial molecule in bone development. Am J Hum Genet 2007; 81(5): 906-912.

9 Hao J, Ramachandran A, George A. Temporal and spatial localization of the dentin matrix proteins during dentin biomineralization. J Histochem Cytochem 2009; 57(3): 227-237.

10 George A, Sabsay B, Simonian PA et al. Characterization of a novel dentin matrix acidic phosphoprotein. Implications for induction of biomineralization. J Biol Chem 1993; 268(17): 12624-12630.

11 Ye L, MacDougall M, Zhang S et al. Deletion of dentin matrix protein-1 leads to a partial failure of maturation of predentin into dentin, hypomineralization, and expanded cavities of pulp and root canal during postnatal tooth development. J Biol Chem 2004; 279(18): 19141-19148.

12 Yang W, Lu Y, Kalajzic I et al. Dentin matrix protein 1 gene cis-regulation: use in osteocytes to characterize local responses to mechanical loading in vitro and in vivo. J Biol Chem 2005; 280(21): 20680-20690.

13 Kalajzic I, Braut A, Guo D et al. Dentin matrix protein 1 expression during osteoblastic differentiation, generation of an osteocyte GFP-transgene. Bone 2004; 35(1): 74-82.

14 Rafaelsen SH, Raeder H, Fagerheim AK et al. Exome sequencing reveals FAM20c mutations associated with fibroblast growth factor 23-related hypophosphatemia, dental anomalies, and ectopic calcification. J Bone Miner Res 2013; 28(6): 1378-1385.

(c) (i) (2) This work is licensed under a Creative Commons AttributionBY NC SA NonCommercial-ShareAlike 3.0 Unported License. The images or other third party material in this article are included in the article's Creative Commons license, unless indicated otherwise in the credit line; if the material is not included under the Creative Commons license, users will need to obtain permission from the license holder to reproduce the material. To view a copy of this license, visit http://creativecommons.org/licenses/by-nc-sa/3.0/ 